\title{
Enhancing the detection of laser-excited strain waves via transparent nanolayers
}

\author{
Hao Zhang $\odot{ }^{1,2}$ Alessandro Antoncecchi, ${ }^{2,3}$ Stephen Edward, ${ }^{2,4}$ Paul Planken, ${ }^{2,4}$ and Stefan Witte $\circledast^{2,3, *}$ \\ ${ }^{1}$ School of Artificial Intelligence, Optics and Electronics (iOPEN), Northwestern Polytechnical University, Xi'an 710072, China \\ ${ }^{2}$ Advanced Research Center for Nanolithography, Science Park 106, 1098 XG Amsterdam, Netherlands \\ ${ }^{3}$ Department of Physics and Astronomy, Vrije Universiteit Amsterdam, De Boelelaan 1105, 1081 HV, Amsterdam, Netherlands \\ ${ }^{4}$ Van der Waals-Zeeman Institute, University of Amsterdam, Science Park 904, 1098 XH Amsterdam, Netherlands
}

(Received 20 July 2021; revised 30 September 2021; accepted 26 October 2021; published 9 November 2021)

\begin{abstract}
Light-induced acoustic waves can be used as sensitive probes, providing a pathway toward microscopic imaging and metrology in optically inaccessible media. The ability to detect such waves depends on the interaction of an optical probe pulse with the acoustic waves in the topmost layers of the structure. Therefore, the interplay between optoacoustic coupling and material boundaries, combined with the properties of acoustic waves near free surfaces is of prime importance. Here we show an approach toward optimized optical detection of such laser-excited acoustic waves. We explore the physics underlying this detection, finding that the presence of a free surface actually reduces the optoacoustic interaction, and subsequently enhancing this interaction via adding transparent nanolayers on the free surface. Our work uncovers an important yet rarely explored aspect in optical detection of strain waves via free surfaces and may lead to strategies for signal enhancement in the imaging and characterization of subsurface structures using laser-excited strain waves.
\end{abstract}

DOI: 10.1103/PhysRevB.104.205416

\section{INTRODUCTION}

Ultrafast laser generation and detection of acoustic waves in solids have attracted increasing attention in recent decades. Thomsen et al. have shown that the absorption of a short light pulse by a solid can lead to the generation of an extremely short stress pulse [1]. The absorption of ultrafast laser pulse by a solid can lead to a material heating rate that is only limited by the fundamental electron-phonon coupling process. With this ultrafast heating rate, it is possible to generate acoustic waves of hundreds of gigahertz (hypersound). The frequencies of the sound waves are so high that the so-called picosecond ultrasonics technique is of great scientific value and shows great promise for a number of applications. Examples include nonlinear acoustics and solitons [2-5], acoustic focusing, and shock waves [6,7], as well as laser-induced sound waves for imaging buried nanostructures $[4,8,9]$ and the detection of subsurface alignment gratings in nanolithography [10,11].

Excitation and detection of strain waves in photoacoustics can be realized by pumping the solid with a pump pulse and detecting its optical response by a time-delayed probe pulse: the pump pulse excites a strain pulse propagating into the solid and being scattered/reflected back to an interface. There, it changes the optical properties of the solid which is detected by the probe pulse. Such an interface is usually a free surface, forming the solid/air boundary. It is well-known that the total

\section{*S.Witte@arcnl.nl}

Published by the American Physical Society under the terms of the Creative Commons Attribution 4.0 International license. Further distribution of this work must maintain attribution to the author(s) and the published article's title, journal citation, and DOI. stress vanishes at free surfaces [1,12]. This implies a variation in the acoustic field imposed by the boundary condition, making the properties of acoustic waves and their interactions with the probing light near the free surface of prime importance for their detection. Although such free surfaces are routinely encountered in experiments adopting all-optical generation and detection of acoustic waves, the implications of the presence of a free surface on the detection of the acoustic waves are rarely discussed in the literature.

The free boundary condition requires the total stress to vanish at free surfaces [1]: $\sigma_{\text {total }}=0$. When an ultrashort laser pulse impinges on the surface of a partially absorptive solid such as a metal, this leads to stresses of different origins: (1) Surface heating gives rise to a surface thermal stress following its temperature increase [1,13]: $\sigma_{\mathrm{th}}=-3 B \beta \Delta T_{l}$, where $B$ is the bulk modulus, $\beta$ is the linear expansion coefficient, and $\Delta T_{l}$ is the increase of lattice temperature. (2) Because of the thermal stress, the solid expands in volume, resulting in a quasistatic surface deformation as long as the surface temperature elevation has not been dissipated. This quasistatic lattice deformation is always associated with a quasistatic surface stress $\sigma_{\text {stat }}$ to balance $\sigma_{\text {th }}$. If the heating is adiabatic, there will be no or very little stress wave generated. (3) The absorption of a femtosecond laser pulse results in a swift heating of the solid surface, followed by a prompt surface expansion, leading to not only static surface stress but also propagating stress $\sigma_{\text {prop }}$. From this analysis, in ultrafast photoacoustics, the total stress is the sum of three contributions: $\sigma_{\text {total }}=\sigma_{\text {th }}+\sigma_{\text {stat }}+\sigma_{\text {prop }}$. The free boundary condition then requires that

$$
\sigma_{\text {th }}+\sigma_{\text {stat }}+\sigma_{\text {prop }}=0
$$

at the free surface. Equation (1) describes the situation when the propagating stress has reached the free surface. When the propagating stress leaves the free surface, only the first two 
contributions remain, i.e.,

$$
\sigma_{\text {th }}+\sigma_{\text {stat }}=0 .
$$

Since Eqs. (1) and (2) have to both be satisfied, the result is that $\sigma_{\text {prop }}=0$ at the free surface. Because the propagating strain and the propagating stress must obey the strain-stress relation $\sigma_{\text {prop }}=-(\lambda+2 \mu) s_{\text {prop }}[14]$, it means that the acoustic echo, manifesting itself as the returned propagating strain, is zero at the free surface.

This assertion has an important consequence for the optical detection of strain waves by photoelastic effects. At the very surface of the metal where the optical intensity of the probe pulse is the highest, its contribution to the reflectivity change of the acoustic echo is actually zero. This implies that the detected echo strength depends strongly on the probe penetration depth. The reflectivity change measured in this case only results from the strain waves below the free surface and within the optical penetration depth of the probe pulse, where the probe intensity has already been attenuated. In metals, the probe penetration depth can be extremely short, making the detection of the strain waves via free surfaces less efficient.

We note that although the above analysis indicates a zero propagating strain at the free surface, the surface displacement, however, is maximum when the surface is free. In interferometric [15] or transient grating $[14,16]$ measurements, the photoelastic response induced by the strain pulse is usually measured together with the optical phase change induced by the surface displacement. In those cases, the detection is not necessarily inefficient when the surface is free. When the acoustic waves are measured via the photoelastic effect as variations in the reflected probe intensity, there is no contribution from the surface displacement, as it only changes the phase of the probe pulse [13].

It is the purpose of this paper to experimentally verify the role of a free surface in strain detection by optical means. We present both experimental and theoretical evidence that the presence of a free surface actually reduces the interaction between the probe light and the acoustic waves, and subsequently introducing a method to enhance this interaction without actually changing the probe penetration. In our experiments, we use specially designed samples to separate the effect of a free surface on the strain detection. The discovered effect is further confirmed by comparing the measurements to an advanced numerical model that we have developed earlier [14], with further implementation of the free-boundary conditions, showing excellent agreement to the measured data. This paper elaborates a key aspect in the detection of strain waves at free surfaces by optical means and can lead to strategies for signal enhancement in the imaging and characterization of subsurface nanostructures using laser-excited acoustic waves. In those applications $[8,10]$, the free-surface effect may be explored to enhance the strain detection via the photoelastic effect.

\section{RESULTS}

\section{A. Experimental setup and samples}

Our experiments are based on an ultrafast pump-probe setup originally developed for high-resolution acoustic imaging [8,17]. A simplified schematic of the setup and the layered

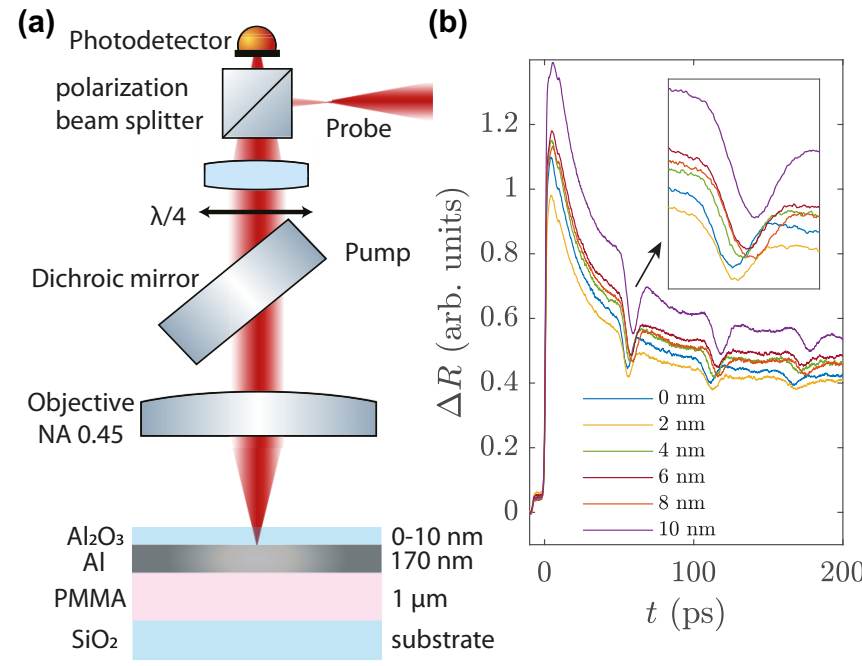

FIG. 1. (a) Simplified schematic of the experimental setup. The pump and probe beams are focused on the surface of the aluminum layer through the $\mathrm{Al}_{2} \mathrm{O}_{3}$ layer by a microscope objective. The dichroic mirror ensures that only the reflected probe beam can reach the photodetector. Samples with $\mathrm{Al}_{2} \mathrm{O}_{3}$ layers of different thicknesses (nominal values of $0-10 \mathrm{~nm}$ ) are used for the experiment. The obtained signal is proportional to the reflectivity change. The measured signals (in arbitrary units) in these samples are shown in (b). The inset in (b) is a magnified plot around the first echo.

samples used in the experiments are shown in Fig. 1(a). The output of a Ti:sapphire oscillator (Femtolasers XL500: wavelength centered at $800 \mathrm{~nm}$, pulse duration $70 \mathrm{fs}$, repetition rate $5.1 \mathrm{MHz}$ ) is split in two beams with a 90:10 beam splitter. The beam with higher intensity is used to generate second harmonic $(400 \mathrm{~nm})$ of the fundamental wavelength using a $\beta$ barium borate (BBO) crystal. This converted $400-\mathrm{nm}$ beam is used as the pump beam with a variable optical path length by using a mechanical translation stage (PI) as the optical delay line. This beam is focused by a microscope objective (Olympus LCPLN20XIR, x20, N.A. 0.45) onto the sample surface. The beam with the fundamental wavelength $(800 \mathrm{~nm})$ is used as the probe beam. The probe light reflected by the sample is focused on a silicon photodiode (Thorlabs PDA100A2). The photodiode signal is sent to a lock-in amplifier referenced by the chopping wheel which modulates the pump beam at a frequency of $5 \mathrm{kHz}$, to increase the signal-to-noise ratio. The experiments are performed on thin aluminum films with a transparent dielectric layer of various thicknesses deposited on their surfaces. The pump and probe beams are focused on the surface of the aluminum layer through the transparent $\mathrm{Al}_{2} \mathrm{O}_{3}$ layer. We have measured the complex refractive index of the $\mathrm{Al}_{2} \mathrm{O}_{3}$ layer by spectroscopic ellipsometry, which shows a negligible imaginary part at both pump and probe wavelengths. The samples were fabricated on glass coverslips (borosilicate $170 \mu \mathrm{m}$ ). A 1- $\mu \mathrm{m}$-thick poly(methyl methacrylate) (PMMA) layer was first deposited on the coverslip by spin coating. The PMMA layer serves to increase the acoustic impedance mismatch at the rear side of the aluminum layer to increase the strength of the reflected strain wave for better detection. The PMMA layer is thick enough to ensure that no acoustic echo from the PMMA/substrate interface is detected 
in our time window of interest. Both the aluminum layer and the $\mathrm{Al}_{2} \mathrm{O}_{3}$ layer were deposited by electron beam physical vapor deposition (E-Flex, Polyteknik). The thickness of the aluminum layer is kept constant for all samples. $\mathrm{Al}_{2} \mathrm{O}_{3}$ layers of various thicknesses $(0,2,4,6,8,10 \mathrm{~nm}$ by nominal values) were deposited on top of the aluminum layers. The dielectric layer repositions the free surface from the metal/air interface to the dielectric/air interface. Thanks to the transparency of the dielectric layer, the probe pulse measures the reflectivity variation always at the metal surface, irrespective of the dielectric layer thickness. The probe light reflected by the sample is collected by a photodiode. The photodiode signal is sent to a lock-in amplifier referenced by a mechanical chopping wheel which modulates the pump beam at a frequency of $5 \mathrm{kHz}$. The setup measures the reflectivity change induced by the excited acoustic wave via the photoelastic effect as a function of the pump-probe delay. A systematic variation of the thickness of the deposited transparent layer results in a reposition of the free surface by a controlled amount such that the effect of the free surface on the strain detection is isolated and systematically studied.

\section{B. Observation of echo enhancement}

Figure 1(b) shows the raw signals measured on these samples. A large and sharp peak is observed immediately after the arrival of the pump pulse which is caused by a swift heating of the free electrons and subsequent heating of the lattice via electron-phonon coupling. Repetitive acoustic echoes are observed, which results from the propagation and reflection of the excited strain pulse in the $\mathrm{Al}_{2} \mathrm{O}_{3}-\mathrm{Al}$ bilayer system. The diminishing of the echo signal after several acoustic round trips is mainly due to acoustic transmission to the PMMA layer and propagation losses.

The magnitude of the aforementioned electron peak and the overall signal strength vary among samples, with a tendency to be larger for samples with a thicker $\mathrm{Al}_{2} \mathrm{O}_{3}$ layer. This tendency can be explained by the difference in the pump beam reflectivities: the sample with a thicker $\mathrm{Al}_{2} \mathrm{O}_{3}$ layer has a smaller reflectivity, thus, an increased absorption of the pump beam. Other experimental uncertainties are likely to cause additional variations in the signal level, such as laser energy fluctuations. To enable a true and fair comparison of the echo strength among samples with different $\mathrm{Al}_{2} \mathrm{O}_{3}$ thicknesses, the data needs to be normalized by the actual absorbed pump energy. Measurements we performed at various pump powers indicate that the magnitude of the electron peak is proportional to the pump power. Therefore, we normalize the signal measured on each sample by its electron peak. The signals after such normalization are shown in Fig. 2(a). It is directly observed that the echo timings shift toward longer time delays as the $\mathrm{Al}_{2} \mathrm{O}_{3}$ thickness is increased, and that the magnitude of the echoes increases. The echo timing shift is caused by an increase of acoustic path length inside the $\mathrm{Al}_{2} \mathrm{O}_{3}$ layer. To quantify these observations, we fit the normalized signal by the combination of an exponential decaying function and multiple Gaussian functions:

$$
\begin{aligned}
\Delta R= & C_{B}+A_{B} e^{\left(-t / \tau_{B}\right)}+A e^{-2\left[\left(t-t_{0}\right) / w_{0}\right]^{2}} \\
& +A_{L} e^{-2\left[\left(t-t_{L}\right) / w_{L}\right]^{2}}+A_{R} e^{-2\left[\left(t-t_{R}\right) / w_{R}\right]^{2}} .
\end{aligned}
$$

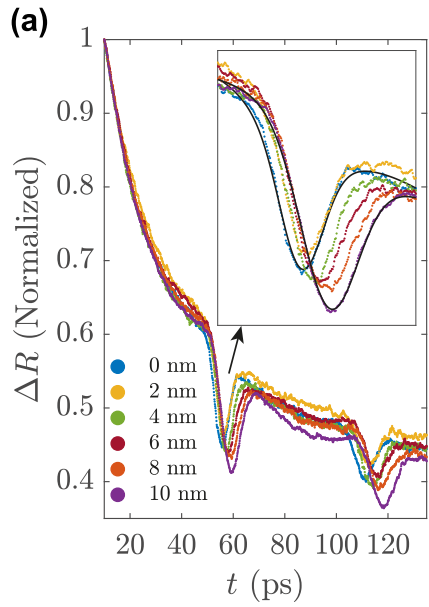

(b)

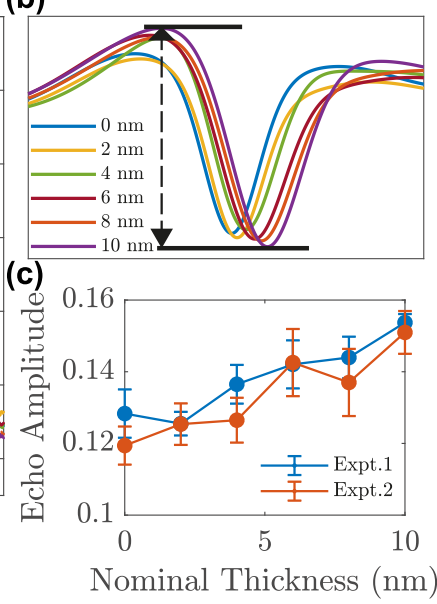

FIG. 2. (a) The measured reflectivity changes after normalization. The data are shown for samples of different $\mathrm{Al}_{2} \mathrm{O}_{3}$ thicknesses, in which their nominal values are labeled. The inset of (a) shows a magnified view around the first echo. The black solid lines in the inset are fits to the measured signal by using Eq. (3). From the fits, the background signals are subtracted, leaving the echoes to be separated and shown in (b). In (b), the echo amplitude is defined as the signal difference between the trough and the first crest in the fit. The extracted amplitudes of the first echo are shown in (c), as a function of nominal thickness of the $\mathrm{Al}_{2} \mathrm{O}_{3}$ layer. The resulting echo amplitudes of two independent experimental runs are shown in (c).

In Eq. (3), the constant $C_{B}$ and the exponential function with amplitude $A_{B}$ describe the background signal. The first Gaussian with an amplitude $A(<0)$ describes the trough part of the echo. The other two Gaussian functions with amplitudes $A_{L}(>0)$ and $A_{R}(>0)$ account for the left and right crests of the echo, which are necessary to accurately fit the tripolar shape of the echoes. Two example function fits to the measured signals by using Eq. (3) are shown as black solid lines in the inset of Fig. 2(a). From the fit, we subtract the background signal, leaving only the contribution from the echo, as shown in Fig. 2(b). The echo amplitude is readily defined as the signal difference between the trough and the first crest in the fit by Eq. (3), as illustrated in Fig. 2(b). We note that the echo amplitudes are determined from the fitted curves, rather than the extracted parameters themselves. The resulting amplitudes of the first echo are plotted in Fig. 2(c), in which the total length of the error bar is equal to two times the standard deviation of five experimental scans performed on the same sample spot. The results of another five experimental scans performed on a different sample spot are also shown (labeled as Expt. 2). From this analysis, it is evident that the echo amplitude correlates with the $\mathrm{Al}_{2} \mathrm{O}_{3}$ thickness.

\section{Theoretical model}

To understand the observed correlation between the echo amplitude and the $\mathrm{Al}_{2} \mathrm{O}_{3}$ thickness, we simulate the experiments by using an advanced numerical model we developed earlier [14]. The model includes the relevant physics in the generation, propagation, and detection of strain waves in metals by ultrafast laser pulses, with the implementation of free 
boundary conditions. In the generation part of the model, we use the Beer-âLambert law to describe the optical intensity inside the metal. The reflectivity variation among samples with different $\mathrm{Al}_{2} \mathrm{O}_{3}$ thicknesses is taken into account by the transfer matrix method [18]. Having the optical intensity inside the metal, we then calculate the lattice temperature elevation induced by the absorption of the laser pulse via the two-temperature model, which is used as a source term in solving the following equation of motion for the acoustic wave $[14,19,20]$ :

$$
\begin{aligned}
\rho \frac{\partial^{2} \mathbf{u}}{\partial t^{2}}=\mu \nabla^{2} \mathbf{u} & +(\mu+\lambda) \nabla(\nabla \cdot \mathbf{u})+\nabla \sigma_{\text {th }} \\
& +\xi \nabla^{2} \mathbf{v}+\left(\xi+\lambda_{v}\right) \nabla(\nabla \cdot \mathbf{v}),
\end{aligned}
$$

where $\sigma_{\text {th }}$ is the thermal stress generated by the laser pulse, $\mathbf{u}$ is the displacement vector, $\rho$ the mass density, $\lambda$ and $\mu$ are the two Lamé parameters, $\mathbf{v}$ is the velocity vector, $\xi$ the coefficient of shear viscosity, and $\lambda_{v}$ is linked to the coefficient of bulk viscosity $\eta$ as $\lambda_{v}=\eta-2 \xi / 3$. The thermal stress $\sigma_{\text {th }}$ is modeled through thermoelasticity $[1,21]$ as $\sigma_{\mathrm{th}}=-3 B \beta \Delta T_{l}$, where $\Delta T_{l}$ is the increase of lattice temperature calculated in the generation step. The third step is to calculate the reflectivity change. For this, we relate the change of dielectric constant to the generated strain by the photoelastic effect [22],

$$
\Delta \epsilon=-\epsilon_{r}^{2} P_{12} s_{x},
$$

where $\epsilon_{r}$ is the dielectric constant of aluminum in the unperturbed state, $P_{12}$ is the complex photoelastic constant for longitudinal waves, and $s_{x}$ is the longitudinal volumetric strain. Throughout the paper, $x$ represents the axis perpendicular to the sample surface while $y$ represents the axis parallel to it. Having the value of the dielectric constant change, the probe reflectivity change is calculated by the transfer matrix method [18], which approximates the spatial variation in the refractive index profile by a stratified medium. The probe reflectivity calculation is performed for every pump-probe delay to compare with the experiment. In the calculation of the dielectric function [Eq. (5)], we have neglected the contributions to the reflectivity change from the shear $\left(s_{x y}\right)$ and the lateral $\left(s_{y}\right)$ strains [17], leaving only the contribution of $s_{x}$ to be included. This is justified by comparing the result to a calculation with those contributions included, which shows a nearly identical ( $<1 \%$ variation) reflectivity change for the first two echoes. Equation (4) is numerically solved by the finite-difference time-domain method with the incorporation of free boundary conditions. More details on the model can be found in Refs. [14,17].

The calculated reflectivity change can be directly compared to our measured signals. We use the model to least-squares fit the signals by minimizing the combined residual on all samples. The electron-phonon coupling constant determines the rate of electron-phonon energy exchange, thus, it influences the frequencies of the generated strain pulse $[14,17]$. It is known that for aluminum, the thermo-optic effect has a strong influence on the background signal [14,17], as the $800 \mathrm{~nm}$ probe wavelength coincides with the peak of aluminum's thermoreflectance spectrum [23]. The value of the photoelastic constant $P_{12}$ influences the observed echo shape. Therefore, these parameters are treated as fitting parameters.
For $P_{12}$, we use our determined value $P_{12}=-0.17-0.012 i$ from previous experiments on aluminum [17], keeping the ratio of the real and imaginary parts unchanged and only allowing its amplitude to slightly change in the fit. The longitudinal speed of sound in aluminum $c_{l}=6420 \mathrm{~m} / \mathrm{s}$ [24] and experimental conditions such as the pump fluence $\left(90 \mathrm{~J} / \mathrm{m}^{2}\right)$ are fixed during the fit.

\section{Signal interpretation}

It is well-known that aluminum quite easily oxidizes when exposed to air [26]. A thicker deposited $\mathrm{Al}_{2} \mathrm{O}_{3}$ layer acts as a protective layer, preventing further natural oxidation. However, for samples with very thin or without deposited $\mathrm{Al}_{2} \mathrm{O}_{3}$ layers, we expect different degrees of natural oxidation to still occur, which will further increase the $\mathrm{Al}_{2} \mathrm{O}_{3}$ layer thickness and decrease the metal layer thickness. Therefore, the actual $\mathrm{Al}_{2} \mathrm{O}_{3}$ and metal layer thicknesses are expected to deviate from their nominal values in the actual experiments. To take those effects into account, individual $\mathrm{Al}_{2} \mathrm{O}_{3}$ layer thicknesses and metal layer thicknesses are also treated as fitting parameters. We expect that our model is able to independently extract both the aluminum and the $\mathrm{Al}_{2} \mathrm{O}_{3}$ layer thicknesses, as they contribute to the signal in different ways: The echo amplitude is primarily determined by the $\mathrm{Al}_{2} \mathrm{O}_{3}$ layer thickness, while both the $\mathrm{Al}_{2} \mathrm{O}_{3}$ and the aluminum layer thicknesses determine the echo timing.

Figure 3(a) shows the measured data and corresponding model fits. The calculated reflectivity, shown as solid lines, reproduces the measurements for all samples well. The best-fit $\mathrm{Al}_{2} \mathrm{O}_{3}$ layer thicknesses are shown in the legend in Fig. 3(a). To confirm the accuracy of the best-fit $\mathrm{Al}_{2} \mathrm{O}_{3}$ thicknesses, we performed independent measurements of the $\mathrm{Al}_{2} \mathrm{O}_{3}$ layer thicknesses by spectroscopic ellipsometry. The model-extracted values and the ellipsometry results are plotted in Fig. 3(c), in which the best-fit values obtained by fitting to the data of an additional experimental run are also shown (labeled by Expt. 2). The model-extracted $\mathrm{Al}_{2} \mathrm{O}_{3}$ layer thicknesses are slightly different than the ellipsometry results. Their difference is about $1.2 \mathrm{~nm}$ on average, which demonstrates the accuracy in the determination of the $\mathrm{Al}_{2} \mathrm{O}_{3}$ layer thickness by our model fit. In addition, the best-fit thicknesses to the two independent experimental runs are consistent within $1 \mathrm{~nm}$.

Ellipsometry does not allow access to the aluminum layer thickness as aluminum is optically opaque. To have an independent measurement of the aluminum layer thickness, we take the ellipsometry results of the $\mathrm{Al}_{2} \mathrm{O}_{3}$ layer thickness as model inputs and only fit the aluminum layer thickness to our measured data. In this way, the aluminum layer thickness is solely determined by the echo timing, which is expected to yield an aluminum layer thickness with a comparable accuracy as the ellipsometry measurement. The thicknesses obtained in this way are labeled as ellipsometry+model fit in Fig. 3(d) and they are plotted along with the purely modelextracted values. Those thickness values are consistent within $2 \mathrm{~nm}$. This demonstrates that our acoustic approach can yield the thickness of both transparent and opaque layers, with close-to-ellipsometry accuracy. In addition, the determined aluminum layer thicknesses are consistent with our expec- 

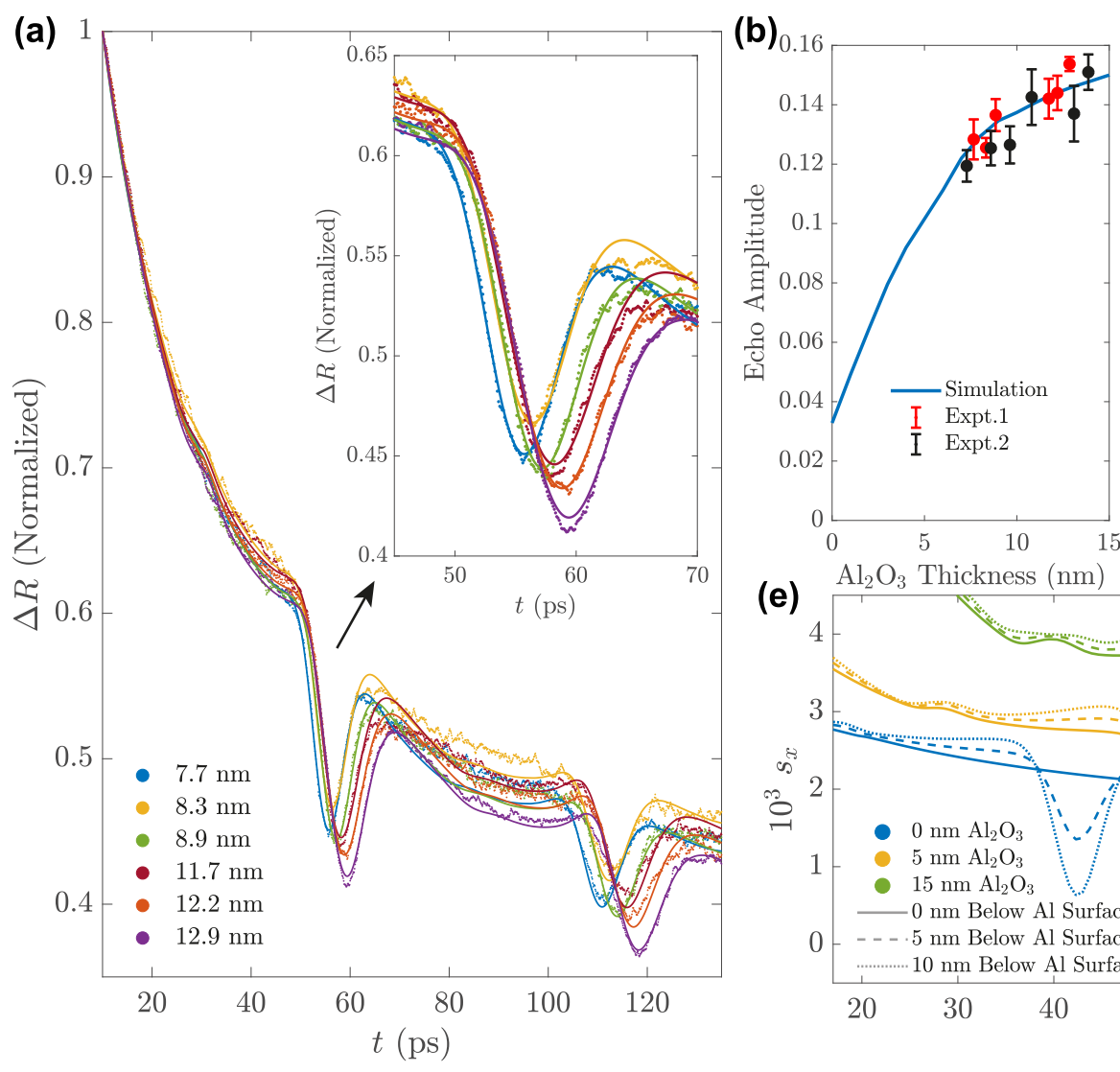

(c)

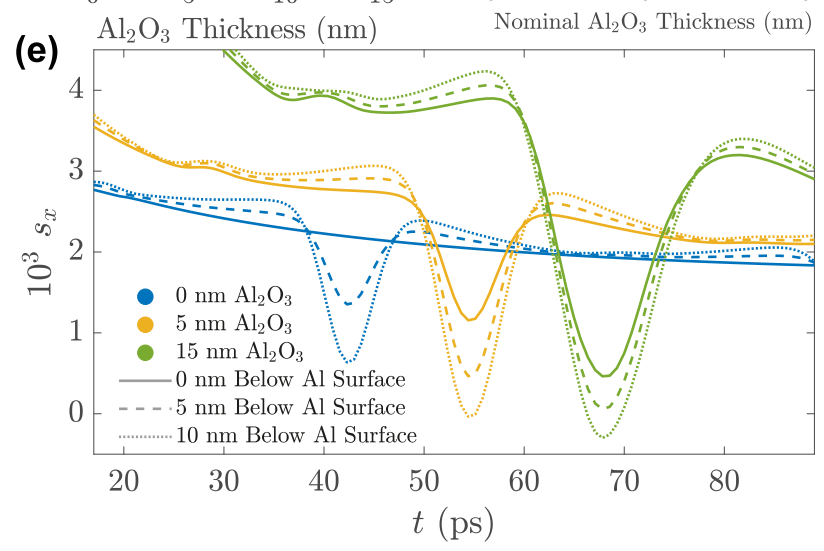

FIG. 3. (a) Model fit to the measurements. The color represents results on samples with different $\mathrm{Al}_{2} \mathrm{O}_{3}$ layer thickness. The dots are the measured data, the solid lines with the same color are the data of the corresponding model fit. The legends in (a) are the best-fit $\mathrm{Al}_{2} \mathrm{O}_{3}$ layer thicknesses. The insets show magnified views around the first echoes. (b) Comparison of the (first) echo amplitude between simulations (solid line) and measurements (dots) versus $\mathrm{Al}_{2} \mathrm{O}_{3}$ layer thickness. The best-fit $\mathrm{Al}_{2} \mathrm{O}_{3}$ layer thicknesses and $\mathrm{Al}$ layer thicknesses are plotted in (c) and (d), respectively, along with the thicknesses measured by ellipsometry. The dynamics of longitudinal strain $\left(s_{x}\right)$ at and below the aluminum surface are shown in (e). The color represents the $\mathrm{Al}_{2} \mathrm{O}_{3}$ layer thickness, while the line style represents the depth where the strain data is shown. Note that the blue and green lines are horizontally shifted by $\mp 10$ ps for clarity.

tations from natural oxidation: For the samples with thinner predeposited $\mathrm{Al}_{2} \mathrm{O}_{3}$ layers, the aluminum layer thickness is smaller, indicating a larger degree of natural oxidation while for the samples with thicker predeposited $\mathrm{Al}_{2} \mathrm{O}_{3}$ layers, the aluminum layer thicknesses saturate at the original value due to the protective effects of a thicker pre-deposited $\mathrm{Al}_{2} \mathrm{O}_{3}$ layer.

The echo amplitudes extracted from the measured and simulated signals are shown in Figs. 3(b). We note that due to natural oxidation, aluminum samples with very thin or no oxide layers are not accessible in our conditions. Yet, for other metals that hardly oxidize naturally, the echo enhancement by depositing a transparent layer is expected to be stronger, as shown by the theoretical curve for thinner transparent layer thicknesses.

The increase in echo amplitude is consistent with the simulated strain values at and below the aluminum surface for samples with different $\mathrm{Al}_{2} \mathrm{O}_{3}$ thickness. These simulated strain dynamics are shown in Fig. 3(e): The echoes are stronger for the samples with a thicker $\mathrm{Al}_{2} \mathrm{O}_{3}$ layer, at all depths. Note the absence of echoes at the aluminum surface in the sample without any $\mathrm{Al}_{2} \mathrm{O}_{3}$ layer is consistent with our earlier conclusion from Eqs. (1) and (2). In addition, the strain profile for a larger $\mathrm{Al}_{2} \mathrm{O}_{3}$ thickness is broader, which is in agreement with the experimental observations. This is caused by strain waves that propagate into the $\mathrm{Al}_{2} \mathrm{O}_{3}$ layer. At the wavelength of our pump beam, the strain waves are not directly generated in the transparent $\mathrm{Al}_{2} \mathrm{O}_{3}$ layer. The energy of the pump pulse is all absorbed in the aluminum layer, causing lattice disturbance at the $\mathrm{Al} / \mathrm{Al}_{2} \mathrm{O}_{3}$ interface. This launches two acoustic pulses propagating in the opposite direction, one into the aluminum and the other one into the $\mathrm{Al}_{2} \mathrm{O}_{3}$. Since the $\mathrm{Al}_{2} \mathrm{O}_{3}$ layer thicknesses are smaller than the acoustic pulse length, the two acoustic pulses do not appear to be separated in our pump-probe trace but appear to be one broader echo.

The propagation of strain waves in the $\mathrm{Al}_{2} \mathrm{O}_{3}$ layer can change its refractive index. However, we estimated this effect and found it to be much smaller than the reflectivity variations caused by the strain propagation in the aluminum layer: the magnitude of the reflectivity changes in our experiments is on the order of $10^{-3}$. To reach the same reflectivity change, but through the $\mathrm{Al}_{2} \mathrm{O}_{3}$ layer alone, we calculated that its refractive index needs to be changed by $2 \%$, for a $10 \mathrm{~nm} \mathrm{Al}{ }_{2} \mathrm{O}_{3}$ layer. Considering that the strain magnitude in our experiments is on the order of $10^{-3}$ and assuming the same strain magnitude as in the aluminum layer, it translates to an unrealistically high strain-optic coefficient of $\frac{d n}{d s_{x}}=20$, while the true value 
(a)

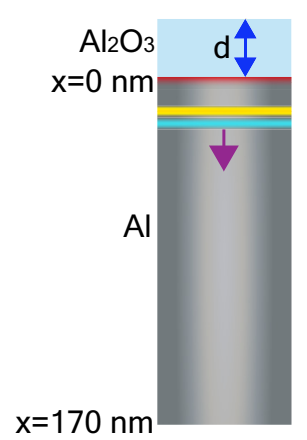

(b)

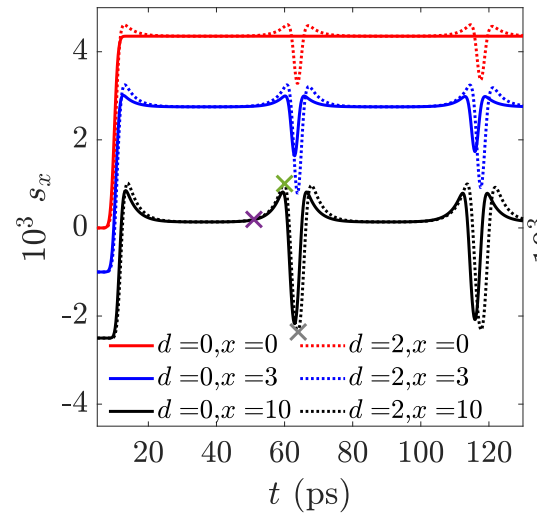

(c)

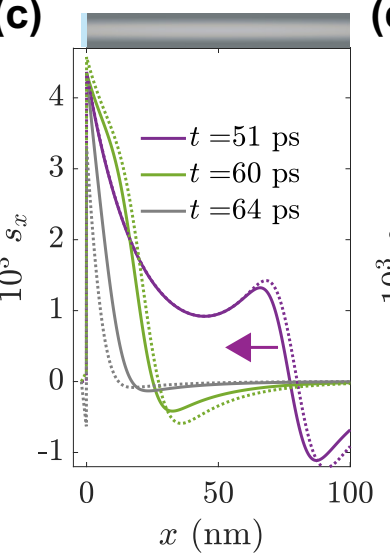

(d)

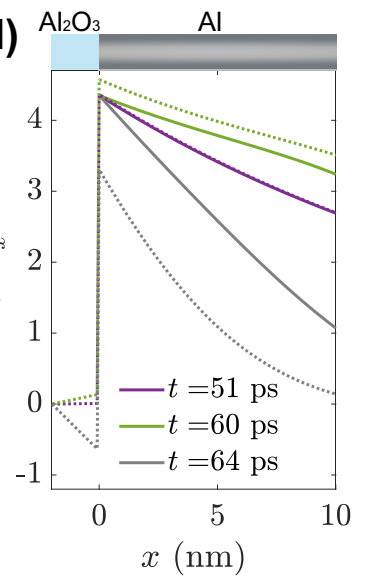

FIG. 4. Propagation of an idealized 1D strain pulse subjected to the free boundary condition, in a $170 \mathrm{~nm}$ aluminum free-standing membrane with a $\mathrm{Al}_{2} \mathrm{O}_{3}$ layer of thickness $d$ on top. (a) A schematic of the sample used in calculations, in which the topmost layers of the aluminum are heated and a strain pulse is launched into the interior. The red region at the aluminum surface illustrates the heat source. The yellow and blue-green area illustrate the positive and negative parts of the generated strain. The purple arrows indicate the propagation direction of the strain. (b) The strain dynamics at three specific positions ( $x=0 \mathrm{~nm}, x=3 \mathrm{~nm}$, and $x=10 \mathrm{~nm}$ ) beneath the aluminum surface. The legends are all in units of $\mathrm{nm}$. Note that the blue and black curves have vertical offsets $\left(-1 \times 10^{3}\right.$ and $-2.5 \times 10^{3}$, respectively) for clarity. (c) The strain spatial profiles at different time instants. The time instants are marked by the three crosses in (b). (d) A zoom-in of (c) in the surface region. In all figures, the solid lines represent calculations without the $\mathrm{Al}_{2} \mathrm{O}_{3}$ layer, the dotted lines represent calculations with a $2 \mathrm{~nm} \mathrm{Al} \mathrm{O}_{3}$ layer on top. See Video 1 in the Supplemental Material for the simulated strain propagation with and without the $\mathrm{Al}_{2} \mathrm{O}_{3}$ layer [25].

should be on the order of unity. Therefore, this effect is negligible in our conditions.

The acoustic wave propagation in the $\mathrm{Al}_{2} \mathrm{O}_{3}$ layer may result in another effect: It may dynamically change the $\mathrm{Al}_{2} \mathrm{O}_{3}$ layer thickness, which in turn leads to reflectivity variations due to the interference of the probe pulse reflections at the top and the bottom interfaces [27]. We have estimated this effect to be much smaller than the photoelastic effect of the aluminum layer. By using the model, we calculate the displacement difference at the top and rear interfaces of the $\mathrm{Al}_{2} \mathrm{O}_{3}$ layer to be around $3 \mathrm{pm}$. We then calculate the reflectivity variation by a change of $3 \mathrm{pm}$ in the $\mathrm{Al}_{2} \mathrm{O}_{3}$ layer thickness, which shows a reflectivity variation on the order of $10^{-6}$, three orders of magnitude smaller than the estimated reflectivity change in our experiments. Therefore, this contribution is neglected in our analysis.

\section{DISCUSSION}

To elaborate the effect of free surface on the strain dynamics, we show the propagation of a hypothetical idealized 1D strain, subjected to the free boundary condition. We note that the purpose of this simulation is not to mimic exact experimental conditions and sample composition/geometry but to conceptually clarify the effect of free-surface effects on strain propagation. The rigorous simulation which takes all of these into account has already been shown to reproduce the experimental signals (Fig. 3). The strain pulse is launched in the simulation by a hypothetical heat source localized at the surface region of a $170 \mathrm{~nm}$ aluminum free-standing membrane. The heat source is described by a swift temperature increase with a rise time of $5 \mathrm{ps}$, with a spatial profile following an exponential decay with a characteristic length of $20 \mathrm{~nm}$ and a maximum temperature increase of $30 \mathrm{~K}$. The heat source is more localized to the surface than that of the rigorous simulation shown before, which ensures a narrow spatial extent of the resulting strain pulse. This results in well-separated echoes and facilitates the observation of free surface effects on strain propagation. In Fig. 4(b), we plot the strain dynamics at three specific locations beneath the aluminum surface, for samples with a $\mathrm{Al}_{2} \mathrm{O}_{3}$ layer of two different thicknesses on top. For the sample without the $\mathrm{Al}_{2} \mathrm{O}_{3}$ layer $(d=0 \mathrm{~nm})$, at the aluminum surface $(x=0 \mathrm{~nm})$, as shown by the solid red trace, only the nonpropagating strain is nonzero. The acoustic echo, caused by the return of the propagating strain, is always zero at the surface. The amplitude of the propagating strain increases as it propagates away from the surface, as shown by the blue and black traces. In contrast, for the sample with a nonzero $\mathrm{Al}_{2} \mathrm{O}_{3}$ layer $(d=2 \mathrm{~nm})$ on top, the propagating strain is nonzero even at the aluminum surface. In addition, the echoes beneath the aluminum surface are all amplified.

The effect of the free surface on the spatial profile of the strain can be seen in Figs. 4(c) and 4(d), where we plot the strain profiles at three time instants. In the solid purple trace of $t=51 \mathrm{ps}$, the propagating strain is separated from the surface strain (quasistatic), and the free boundary condition at this time instant is written as Eq. (2). In the solid green and gray traces at later times, the propagating strain arrives at the free surface and overlaps with the surface strain, and the free boundary condition is written as Eq. (1). Because of those boundary conditions, the strain at the aluminum surface remains the same and is not affected by the return of the propagating strain. This can be clearly seen in Fig. 4(d), where the strain at the aluminum surface $(x=0 \mathrm{~nm})$ remains the same at all times for the sample without the $\mathrm{Al}_{2} \mathrm{O}_{3}$ layer (solid lines). In contrast, the returning of the strain in the sample with a nonzero $\mathrm{Al}_{2} \mathrm{O}_{3}$ layer does alter the strain at the aluminum surface and amplifies the echoes at all depths (dotted lines). The 
transparent $\mathrm{Al}_{2} \mathrm{O}_{3}$ layer repositions the free boundary away from the detection surface, enables the probe pulse to sample a nonzero strain wave even at the metal surface, and amplifies the echoes below it, resulting in an enhanced detection of the acoustic echoes. In addition to our rigorous simulation of the experimental signals discussed earlier, these simulations of an idealized situation provide an intuitive picture and explain the increase of echo amplitude observed in our experiments.

\section{SUMMARY}

The effect of a free surface on strain detection was systematically studied by ultrafast pump-probe generation and detection of acoustic waves. The deposition of $\mathrm{Al}_{2} \mathrm{O}_{3}$ nanolayers of various thicknesses on top of a thin aluminum layer allows the separation of free-surface effect on strain detection. The measured echo amplitude shows a strong correlation with the $\mathrm{Al}_{2} \mathrm{O}_{3}$ layer thickness, which is excellently reproduced by advanced numerical modeling of the generation, propagation, and detection of laser-excited strain waves on free surfaces. It was shown that the presence of a free surface actually prevents the probe pulse from detecting the full strength of the excited acoustic echoes and the detection can be enhanced by adding transparent nanolayers on the free surface. This paper unravels the importance of free-surface effect on strain detection and provides a strategy to enhance the detection of laser-excited strain waves via free surfaces.

\section{ACKNOWLEDGMENTS}

This research was supported by the European Research Council (ERC Starting Grant No. 637476) and the Netherlands Organization for Scientific Research (NWO). The Advanced Research Center for Nanolithography (ARCNL) is a public-private partnership of the University of Amsterdam (UvA), the Vrije Universiteit Amsterdam (VU), NWO and the semiconductor equipment manufacturer ASML.
[1] C. Thomsen, H. T. Grahn, H. J. Maris, and J. Tauc, Surface generation and detection of phonons by picosecond light pulses, Phys. Rev. B 34, 4129 (1986).

[2] H.-Y. Hao and H. J. Maris, Experiments with acoustic solitons in crystalline solids, Phys. Rev. B 64, 064302 (2001).

[3] O. L. Muskens and J. I. Dijkhuis, High Amplitude, Ultrashort, Longitudinal Strain Solitons in Sapphire, Phys. Rev. Lett. 89, 285504 (2002).

[4] B. C. Daly, N. C. R. Holme, T. Buma, C. Branciard, and T. B. Norris, Imaging nanostructures with coherent phonon pulses, Appl. Phys. Lett. 84, 5180 (2004).

[5] P. J. S. van Capel and J. I. Dijkhuis, Time-resolved interferometric detection of ultrashort strain solitons in sapphire, Phys. Rev. B 81, 144106 (2010).

[6] J. H. Lopes, M. A. B. Andrade, J. P. Leao-Neto, J. C. Adamowski, I. V. Minin, and G. T. Silva, Focusing Acoustic Beams with a Ball-Shaped Lens Beyond the Diffraction Limit, Phys. Rev. Appl. 8, 024013 (2017).

[7] B. Giammarinaro, D. Espindola, F. Coulouvrat, and G. Pinton, Focusing of Shear Shock Waves, Phys. Rev. Appl. 9, 014011 (2018).

[8] A. Antoncecchi, H. Zhang, S. Edward, V. Verrina, P. C. M. Planken, and S. Witte, High-resolution microscopy through optically opaque media using ultrafast photoacoustics, Opt. Express 28, 33937 (2020).

[9] K.-H. Lin, C.-T. Yu, S.-Z. Sun, H.-P. Chen, C.-C. Pan, J.-I. Chyi, S.-W. Huang, P.-C. Li, and C.-K. Sun, Two-dimensional nanoultrasonic imaging by using acoustic nanowaves, Appl. Phys. Lett. 89, 043106 (2006).

[10] S. Edward, H. Zhang, I. Setija, V. Verrina, A. Antoncecchi, S. Witte, and P. Planken, Detection of Hidden Gratings Through Multilayer Nanostructures Using Light and Sound, Phys. Rev. Appl. 14, 014015 (2020).

[11] V. Verrina, S. Edward, H. Zhang, S. Witte, and P. C. M. Planken, Photoacoustic detection of low duty cycle gratings through optically opaque layers, Appl. Phys. Lett. 117, 051104 (2020).
[12] B. A. Auld, Acoustic Fields and Waves in Solids (Wiley, New York, 1973), Vol. 1.

[13] O. Matsuda, M. C. Larciprete, R. L. Voti, and O. B. Wright, Fundamentals of picosecond laser ultrasonics, Ultrasonics 56, 3 (2015).

[14] H. Zhang, A. Antoncecchi, S. Edward, I. Setija, P. Planken, and S. Witte, Unraveling Phononic, Optoacoustic, and Mechanical Properties of Metals with Light-Driven Hypersound, Phys. Rev. Appl. 13, 014010 (2020).

[15] O. Matsuda, M. Tomoda, T. Tachizaki, S. Koiwa, A. Ono, K. Aoki, R. P. Beardsley, and O. B. Wright, Ultrafast ellipsometric interferometry for direct detection of coherent phonon strain pulse profiles, J. Opt. Soc. Am. B 30, 1911 (2013).

[16] T. F. Crimmins, A. A. Maznev, and K. A. Nelson, Transient grating measurements of picosecond acoustic pulses in metal films, Appl. Phys. Lett. 74, 1344 (1999).

[17] H. Zhang, A. Antoncecchi, S. Edward, P. Planken, and S. Witte, Ultrafast laser-induced guided elastic waves in a freestanding aluminum membrane, Phys. Rev. B 103, 064303 (2021).

[18] M. Born and E. Wolf, Principles of Optics (Cambridge University Press, Cambridge, 1999).

[19] P. M. Shearer, Introduction to Seismology (Cambridge University Press, New York, 2009).

[20] R. D. Blandford and K. S. Thorne, Applications of Classical Physics (Stanford University and California Institute of Technology, Pasadena, 2013).

[21] O. B. Wright, Ultrafast nonequilibrium stress generation in gold and silver, Phys. Rev. B 49, 9985 (1994).

[22] O. Matsuda, O. B. Wright, D. H. Hurley, V. Gusev, and K. Shimizu, Coherent shear phonon generation and detection with picosecond laser acoustics, Phys. Rev. B 77, 224110 (2008).

[23] R. B. Wilson, B. A. Apgar, L. W. Martin, and D. G. Cahill, Thermoreflectance of metal transducers for optical pumpprobe studies of thermal properties, Opt. Express 20, 28829 (2012).

[24] D. R. Lide, CRC Handbook of Chemistry and Physics, Internet Version 2005 (CRC Press, Boca Raton, FL, 2005). 
[25] See Supplemental Material at http://link.aps.org/supplemental/ 10.1103/PhysRevB.104.205416 for a video showing the simulated strain propagation.

[26] J. Evertsson, F. Bertram, F. Zhang, L. Rullik, L. Merte, M. Shipilin, M. Soldemo, S. Ahmadi, N. Vinogradov, F. Carlà, J. Weissenrieder, M. Göthelid, J. Pan, A. Mikkelsen, J.-O.
Nilsson, and E. Lundgren, The thickness of native oxides on aluminum alloys and single crystals, Appl. Surf. Sci. 349, 826 (2015).

[27] A. Devos, J.-F. Robillard, R. Cote, and P. Emery, High-laserwavelength sensitivity of the picosecond ultrasonic response in transparent thin films, Phys. Rev. B 74, 064114 (2006). 